\title{
Hypoxia of PC-3 prostate cancer cells enhances migration and vasculogenesis in vitro of bone marrow-derived endothelial progenitor cells by secretion of cytokines
}

\author{
SHUAI HUANG ${ }^{1 *}$, LONGYUN PENG $^{2 *}$, YUBO TANG $^{1}$, LONGJUAN ZHANG $^{3}$, \\ WEI GUO $^{1}$, XUENONG ZOU ${ }^{1}$ and XINSHENG PENG ${ }^{1}$ \\ Departments of ${ }^{1}$ Orthopaedic Surgery/Orthopaedic Research Institute and ${ }^{2}$ Cardiology; ${ }^{3}$ The Surgical Laboratory, \\ The First Affiliated Hospital of Sun Yat-sen University, Guangzhou, Guangdong 510080, P.R. China
}

Received November 5, 2012; Accepted February 22, 2013

DOI: 10.3892/or.2013.2363

\begin{abstract}
Hypoxia is a key inducer of neovascularization which is essential for tumor growth, invasion and metastasis. It has been proposed that the recruitment of bone-marrowderived endothelial progenitor cells (BM-EPCs) is pivotal and requires the participation of several tumor-derived cytokines. However, it is not known whether prostate cancer (PCa) cells contribute to the recruitment and vasculogenesis of EPCs in PCa progression. In the present study, we demonstrated that all conditioned medium (CM) of PC-3 PCa cells promoted proliferation and migration, and augmented the vasculogenesis capacity of BM-EPCs, and 24-h hypoxia (24H)-CM presented stronger ability compared to $24-\mathrm{h}$ normoxia (24N)-CM and 48H-CM. Human cytokine antibody array with 174 anticytokine antibodies revealed the changes of cytokine in CMs. Twenty-five types of cytokines significantly increased in $24 \mathrm{H}-\mathrm{CM}$ compared with $24 \mathrm{~N}-\mathrm{CM}$. Eleven types of cytokines (5 factors increased and 6 decreased) were significantly different between $48 \mathrm{H}-\mathrm{CM}$ and $48 \mathrm{~N}-\mathrm{CM}$. Twelve types of cytokines (4 factors increased and 8 decreased) were signifi-
\end{abstract}

Correspondence to: Professor Xinsheng Peng, Department of Orthopaedic Surgery, The First Affiliated Hospital of Sun Yat-sen University, 58 Zhongshan 2rd Road, Guangzhou, Guangdong 510080, P.R. China

E-mail: pengxs66@yahoo.com

*Contributed equally

Abbreviations: PCa, prostate cancer; Hx, hypoxia; Nx, normoxia; BM-EPC, bone marrow-derived endothelial progenitor cell; CM, conditioned medium; H-CM, hypoxic conditioned medium; N-CM, normoxic conditioned medium; NM, normal medium; 24N, 24-h normoxia; $24 \mathrm{H}, 24-\mathrm{h}$ hypoxia; 48N, 48-h normoxia; 48H, 48-h hypoxia

Key words: bone marrow-derived endothelial progenitor cell, prostate cancer cell, hypoxia, cytokine, migration, vasculogenesis cantly different between 48H-CM and 24H-CM. Furthermore, according to the Gene Ontology analysis, all altered cytokines were involved in proliferation, chemotaxis, cell motility, cell migration, vasculogenesis and angiogenesis. Of note, the changed regularity of cytokines in the $24 \mathrm{H}-\mathrm{CM}$ and $48 \mathrm{H}-\mathrm{CM}$ of PC-3 cells was in concert with the functional changes of BM-EPCs treated by different CM of PC-3 cells in enhancing the proliferation, migration and vasculogenesis potential of BM-EPCs. These findings suggest that PCa cells may have the potential to modulate their microenvironment and facilitate BM-EPC migration and vasculogenesis by secretion of cytokines in the early stage of hypoxia.

\section{Introduction}

Bone is the most common site of prostate cancer (PCa) metastasis and once tumors metastasize to bone, they are essentially incurable and result in significant morbidity prior to patient mortality $(1,2)$. Therefore, to develop more effective therapeutics, a more thorough understanding of the key complex processes that are central to metastasis may reveal more robust and less redundant therapeutic targets.

Neovascularization is essential for tumor growth and it also facilitates tumor invasion and metastasis (3). Furthermore, in cancer patients with metastasis, neovascularization-mediated progression of micrometastasis to lethal macrometastasis is the major cause of mortality (4). Emerging evidence implicates hypoxia as a key inducer of neovascularization in tumors (5). Moreover, hypoxia-induced pathological neovascularization mediates tumor cell dissemination, invasion and metastasis in an animal model (6). However, the mechanisms underlying pathological neovascularization in relation to hypoxia in tumor invasion and metastasis remain elusive.

The process of hypoxia-induced neovascularization was formerly attributed to the migration and proliferation of preexisting, fully differentiated endothelial cells (ECs), known as angiogenesis (7). However, it is now well established that tumors can acquire their vasculature by various mechanisms including postnatal vasculogenesis, a process during which circulating bone-marrow-derived endothelial progenitor cells (BM-EPCs) home to sites of neovascularization and diffe- 
rentiate into ECs (8). Although there has been controversy concerning the time during neovascularization at which the proposed contribution of EPCs to neovascular vessels might occur (9), it has been proposed that the recruitment of BM-EPCs is pivotal for tumor vasculogenesis (10-12). Notably, recent studies have shown that BM-EPCs are recruited to the angiogenic switch in tumor growth and metastatic progression $(4,13,14)$ and blood levels of EPCs tend to increase in cancer patients and correlate with the stage of the malignant disease (15). In prostate cancer, although microvessel density is related to clinical stage, progression, metastasis and survival (16-18), it is not known whether EPCs contribute to the bone metastasis of PCa.

The mobilization, homing and incorporation of EPCs into tumors are multi-step and multi-factor events during tumor vasculogenesis. This complex process requires the participation of numerous cy tokines. A series of studies have shown that several factors, such as VEGF (20), SDF-1 (20), G-CSF (21), GM-CSF (22), PIGF (23), IGF2 (24), E-selectin (25) and angiopoietin-2 (26), play an important role in inducing EPC homing and vasculogenesis. Although it is not very clear how circulating EPC home from the bone marrow specifically into the tumor microenvironment, the mobilization of EPC from the bone marrow into the bloodstream, migration to the tumor site, and subsequent integration into the vascular network, are all presumably controlled by tumor-derived factors (11). However, in PCa progression, it is not clear whether the above-mentioned tumor-derived factors are involved in the regulation of EPC migration and vasculogenesis in hypoxia, and whether other cytokines play roles in these processes. Since a recent study found that PCa cells which metastasize to bone competed with haematopoietic stem cells for the niche and colonize in the niche (27) and haematopoietic stem cell niches within the bone marrow are hypoxic $(28,29)$, we speculated that PCa cells in the hypoxic microenvironment could recruit $\mathrm{BM}-\mathrm{EPCs}$ and induce vasculogenesis by releasing hypoxia-induced cytokines.

In this in vitro study, we found that conditioned medium (CM) of PC-3 cells, derived from bone metastasis of PCa, by hypoxia for $24 \mathrm{~h}$ promoted proliferation and migration, and augmented the vasculogenesis capacity of BM-EPCs. Human cytokine antibody array revealed that under early hypoxia, the PC-3 cells secreted several cytokines involved in BM-EPC functions, including proliferation, migration and vasculogenesis. These findings suggest that PCa cells may have the potential to modulate their microenvironment and facilitate BM-EPC migration and vasculogenesis in the early stage of hypoxia.

\section{Materials and methods}

Cell culture and hypoxic treatment. PC-3 cells were purchased from the American Type Culture Collection (ATCC, Manassas, VA, USA) and maintained in F-12 culture medium (HyClone) supplemented with $10 \%$ fetal bovine serum (HyClone). For normoxia experiments, the PC-3 cells were cultured at $37^{\circ} \mathrm{C}$ with $5 \% \mathrm{CO}_{2} / 95 \%$ air in a humidified incubator, and for hypoxia experiments, the PC-3 cells were cultured at $37^{\circ} \mathrm{C}$ with $5 \% \mathrm{CO}_{2}, 94 \% \mathrm{~N}_{2}$, and $1 \% \mathrm{O}_{2}$ in a multigas incubator (Juji Field, Tokyo, Japan).
Conditioned medium collection. PC-3 cells were cultured in hypoxia and normoxia for 24 and $48 \mathrm{~h}$ and $\mathrm{CM}$ was collected. Briefly, PC-3 cells were seeded at a density of $2.5 \times 10^{5}$ cells $/ \mathrm{ml}$ in F12 (HyClone) complete media into a 10-cm cell culture dish and grown to $\sim 80 \%$ confluency. Cells were washed twice with phosphate-buffered saline (PBS) and incubated in serum-free medium for 24 and $48 \mathrm{~h}$. CM was collected and centrifuged at $2,000 \mathrm{x} \mathrm{g}$ at $4^{\circ} \mathrm{C}$ for $10 \mathrm{~min}$ to remove cell debris and the supernatant was used.

Isolation and cultivation of BM-EPCs. Bone marrow was collected from male patients with lumbar degenerative diseases (age range, 27-72 years; mean age 52.4+13.9 years; disc herniation with lumbar spondylolisthesis, degenerative lumbar spondylolisthesis and lumbar spinal stenosis with instability) at the time of operation. Informed consent was obtained from the patients for bone marrow blood collection, and all procedures were performed in accordance with the guidance and approval of a research Ethics Committee at the First Affiliated Hospital of Sun Yat-sen University.

Mononuclear cells were collected by density gradient centrifugation using Ficoll-Paque ${ }^{\mathrm{TM}}$ Premium (1.077; GE Healthcare, San Francisco, CA, USA) according to the manufacturer's instructions (30). Briefly, the isolated cells were cultivated in dishes coated with fibronectin and induced by EGM-2 MV Single Quots (Cambrex) at $37^{\circ} \mathrm{C}$ with $5 \% \mathrm{CO}_{2} / 95 \%$ air in a humidified incubator at a density of $3-5 \times 10^{6} / \mathrm{cm}^{2}$. After 3 days in culture, non-adherent cells were removed by washing with new medium and changed. After 7 days in culture, BM-EPCs were identified by immunofluorescence staining and flow cytometry. Quantitative fluorescence-activated cell sorting (FACS) was performed on a FACSVantage SE flow cytometer (Becton-Dickinson, San Jose, CA, USA). The Weibel-Palade body in cultivation cells was visualized by transmission electron microscope (TEM).

BM-EPC proliferation assay. EPC proliferation assay was performed to assess the viability of BM-EPCs treated with $\mathrm{CM}$ of PC-3 cells. The effect of CM on BM-EPC proliferation was determined by 2-(2-methoxy-4-nitrophenyl)-3-(4-nitrophenyl)5-(2,4-disulfophenyl)-2H-tetrazolium, monosodium salt (WST-8) assay kit (CCK-8, Dojindo, Tokyo, Japan). Briefly, BM-EPCs were plated in 96-well plates at a concentration of $5 \times 10^{3}$ cells/well in $100 \mu \mathrm{l} \mathrm{CM}$ of PC-3 cells. Following incubation for $36 \mathrm{~h}$ by CM, CCK- 8 was used according to the manufacturer's instructions. WST- 8 was added into each well for $4 \mathrm{~h}$ prior to the measurement. The absorbance at $450 \mathrm{~nm}$ was measured using a microplate reader.

BM-EPC migration assay. Cell migration was evaluated using the Transwell system (Corning Costar, Acton, MA, USA) with $6.5-\mathrm{mm}$ diameter polycarbonate filters $(8-\mu \mathrm{m}$ pore size). Briefly, BM-EPCs were seeded onto chemotaxis filters in $100 \mu \mathrm{l} \mathrm{F-12} \mathrm{medium.} \mathrm{CM} \mathrm{of} \mathrm{PC-3} \mathrm{cells} \mathrm{cultured} \mathrm{under}$ normoxia and hypoxia for 24 and $48 \mathrm{~h}$ was collected and then added to the lower chamber with a normal medium (NM; F-12) as control medium. After the 24-h migration period, non-migrating cells were completely removed from the top surface of the membrane and attached cells were fixed with $95 \%$ ethanol for $10 \mathrm{~min}$ and stained with $0.1 \%$ crystal violet for 
$25 \mathrm{~min}$. The plate was immersed in fresh tap water to remove excess dye. The adherent cells were visualized under light microscopy at x200 magnification and quantified in triplicate by counting adherent cells in 5 randomly selected fields per well (Axiovert 100; Carl Zeiss Microimaging, Thornwood, NY, USA). The results are representative of 4 independent experiments.

In vitro BM-EPC vasculogenesis assay. In vitro $\mathrm{EPC}$ vasculogenesis assay was performed with In Vitro Angiogenesis Assay kit (Chemicon, Temecula, CA, USA) according to the manufacturer's instructions. Briefly, EC Matrix ${ }^{\mathrm{TM}}$ solution was thawed on ice overnight, mixed with 10X EC Matrix diluents and placed in a 96-well tissue culture plate at $37^{\circ} \mathrm{C}$ for $1 \mathrm{~h}$ to allow the matrix solution to solidify. BM-EPCs were plated in normoxic or hypoxic CM at a concentration of $1 \times 10^{4}$ cells/well on the top of the solidified matrix solution (1\% FBS was added to $\mathrm{CM}$ and $\mathrm{NM}$ ). Tube formation was inspected under an inverted light microscope at x200 magnification. Tube formation was defined as a structure exhibiting a length four times its width. Five independent fields were assessed for each well, and the average number of tubes/x200 field was determined.

Human cytokine antibody array. Cytokine concentrations in $\mathrm{CM}$ were quantified by using human cytokine antibody arrays. RayBio Human Cytokine Antibody Array C Series 2000 kit was purchased from RayBiotech (Norcross, GA, USA). This array consisted of 174 different antibodies spotted in duplicate onto three membranes. $\mathrm{CM}$ was collected from normoxic or hypoxic PC-3 cells for 24 and $48 \mathrm{~h}$ with an NM (F-12) as control medium. Experiments were performed by KangChen Bio-tech (Shanghai, China) as recommended by the manufacturer. The signal intensities can be quantified by densitometry, and relative expression levels of cytokines can be acquired by comparing them. Positive control can be used to normalize the results from different membranes being compared.

Gene Ontology analysis. The Gene Ontology (GO) project provides a controlled vocabulary to describe gene and gene product attributes in any organism (http://www.geneontology. org). The ontology covers three domains: biological process, cellular component and molecular function. Fisher's exact test is used to find if there is more overlap between the DE list and the GO annotation list than would be expected by chance. The $\mathrm{P}$-value denotes the significance of GO terms enrichment in the DE genes. The lower the P-value, the more significant the GO Term (P-value $\leq 0.05$ is recommended). GOID, the ID of the Gene Ontology term used in Gene Ontology Project. Term, the name of the gene Ontology Term. Ontology, the ontology the GOID belongs to, i.e., 'biological process', 'cellular component' or 'molecular function'.

Statistical analysis. The experimental data are presented as the means of each condition \pm SEM. One-way ANOVA was performed for comparing more than two groups, and paired Student's t-test was performed when comparing two conditions (SPSS for Windows ${ }^{\circledR}$ version 16.0). $\mathrm{P}<0.05\left({ }^{*} \mathrm{P}<0.05\right.$, $\left.{ }^{* *} \mathrm{P}<0.01\right)$ was considered to indicate a statistically significant difference.
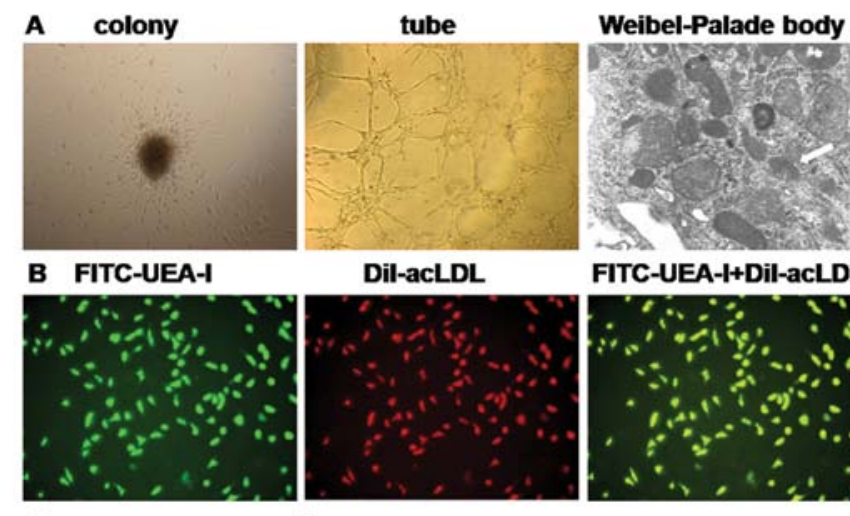

Dil-acLDL

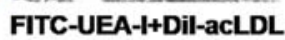

C

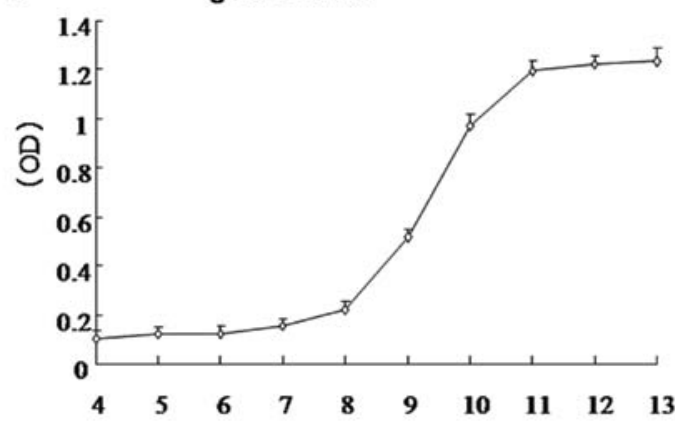

Figure 1. The growth processes of BM-EPCs. (A) The morphology of BM-EPCs in 7-day-cultured BM-EPCs formed colonies, tubes on the ECM in vitro and Weibel-Palade body (arrow) in the endochylema by TEM. (B) Immunofluorescence characterization of BM-EPCs. Binding to FITC-UEA-I, endocytosis of Dil-acLDL, and double positive cells appearing yellow in the overlay were identified as differentiating BM-EPCs. (C) The growth curve of BM-EPCs is shown.

\section{Results}

The growth processes of BM-EPCs. Mononuclear cells were isolated from human bone marrow blood and cultivated in EGM2 on fibronectin-coated dishes. Cells demonstrated round morphology with different sizes as assessed by light microscopy during the first 3 days. With a tendency of $20 \%$ of the mononuclear cells transferred to culture plates grew as adherent cells during 7 days of cultivation and clusters or colonies formed. Furthermore, in endochylema under TEM, the Weibel-Palade body was also observed and BM-EPCs had active angiogenic potential (Fig. 1A). Following cultivation for 7 days, the adherent cells exhibited strong ability to uptake Dil-acLDL, and FITC-UEA-I and the double positive rate was $(94.3 \pm 4.1 \%)$ (Fig. 1B), which were identified as differentiating EPCs. The growth curve is shown in Fig. 1C. The percentage of expression of CD133, CD34, KDR, VE-cadherin, E-selectin and vWF was determined by flow cytometry at Day 7 and Day 14 of culture. The expression pattern of surface markers in BM-EPCs changed toward a more mature endothelial cell phenotype from Day 7 to Day 14 (Table I).

H-CM of PC-3 cells promotes proliferation of BM-EPCs. The proliferation of EPCs, as a crucial step of vasculogenesis, is triggered and modulated by a variety of stimuli including hypoxic microenvironment. To demonstrate whether $\mathrm{H}-\mathrm{CM}$ for 24 and $48 \mathrm{~h}$ promoted proliferation of BM-EPCs, the proliferation assay was performed to assess the viability of 
Table I. The expression pattern of surface markers of BM-EPCs at Day 7 and Day 14.

\begin{tabular}{lcccccc}
\hline Time & CD133 & CD34 & KDR & VE-cadherin & E-selectin & vWF \\
\hline Day 7 & $19.23 \pm 5.0$ & $41.49 \pm 9.01$ & $65.97 \pm 7.2$ & $21.27 \pm 6.9$ & $8.02 \pm 5.53$ & $5.15 \pm 2.72$ \\
Day 14 & $4.04 \pm 3.52$ & $32.94 \pm 8.37$ & $82.57 \pm 6.2$ & $25.23 \pm 4.4$ & $45.08 \pm 7.77$ & $30.3 \pm 7.81$ \\
P-value & 0.013 & 0.295 & 0.039 & 0.448 & 0.003 & 0.002 \\
\hline
\end{tabular}

BM-EPCs treated with CM of tumor cells. WST-8 assay was used to examine the changes in cell proliferation. We exposed BM-EPCs to 24 and $48 \mathrm{H}-\mathrm{CM}, 24 / 48 \mathrm{~N}-\mathrm{CM}$ and NM as a control medium for $36 \mathrm{~h}$. All CM induced BM-EPC proliferation and $24 \mathrm{H}-\mathrm{CM}$ promoted proliferation of BM-EPCs more evidently compared to $24 \mathrm{~N}-\mathrm{CM}$ (Fig. 2). However, $48 \mathrm{~N}-\mathrm{CM}$ promoted proliferation of BM-EPCs more apparently compared to $48 \mathrm{H}-\mathrm{CM}$. Furthermore, $24 \mathrm{H}-\mathrm{CM}$ had stronger effects on enhancing BM-EPC proliferation than 48H-CM. These results suggested that hypoxia induced PC-3 cells to release some factors which promoted the proliferation of BM-EPCs.

H-CM of PC-3 cells promotes migration of BM-EPCs. The mobilization of EPC from the bone marrow into the bloodstream, migration to the tumor site, and subsequent integration into the vascular network are all presumably controlled by tumor-derived factors and those factors are released to the tumor microenvironment that is $\mathrm{CM}$ of tumor cells in vitro. To determine if $\mathrm{CM}$ collected from hypoxia-treated PC-3 cells ( $\mathrm{H}-\mathrm{CM})$ for 24/48 $\mathrm{h}$ increased migration of BM-EPCs compared to CM collected from normoxia-treated PC-3 cells (N-CM), we used a Transwell assay to estimate the ability of EPCs to migrate. All CM enhanced BM-EPC migration compared with NM as a control (Fig. 3A and C). Also, 24H-CM enhanced migration of BM-EPCs more apparently than $24 \mathrm{~N}-\mathrm{CM}$. However, $48 \mathrm{~N}-\mathrm{CM}$ increased migration of BM-EPCs more evidently than $48 \mathrm{H}-\mathrm{CM}$. Moreover, $24 \mathrm{H}-\mathrm{CM}$ enhanced migration of BM-EPCs more apparently than 48H-CM. These observations suggested that hypoxia induced PC-3 cells to release some factors, which promoted the migration of BM-EPCs.

H-CM of PC-3 cells augments in vitro vasculogenesis of $B M-E P C s$. One of the most prominent effects of hypoxia is the induction of tumor neovascularization (5). To determine whether CM of PC-3 cells augmented the vasculogenesis of BM-EPCs in vitro, we exposed BM-EPCs to $24 / 48 \mathrm{H}-\mathrm{CM}$, $24 / 48 \mathrm{~N}-\mathrm{CM}$ and NM as a control medium for $18 \mathrm{~h}$ on Matrigel. All CM induced BM-EPC tube formation (Fig. 3B and D). The 24H-CM augmented the tube formation of BM-EPCs more evidently than $24 \mathrm{~N}-\mathrm{CM}$. However, $48 \mathrm{~N}-\mathrm{CM}$ augmented the tube formation of BM-EPCs more apparently than $48 \mathrm{H}-\mathrm{CM}$. At the same time, 24H-CM had stronger effects on enhancing BM-EPC tube formation than $48 \mathrm{H}-\mathrm{CM}$. These observations indicated that hypoxia induced PC-3 cells to release some factors which augmented the in vitro vasculogenesis capacity of BM-EPCs.

Significant alterations of the cytokines in $\mathrm{H}$-CM. In order to investigate tumor-derived cytokines of $\mathrm{PC}-3$ cells which effect the functions of BM-EPCs, we used human cytokine antibody

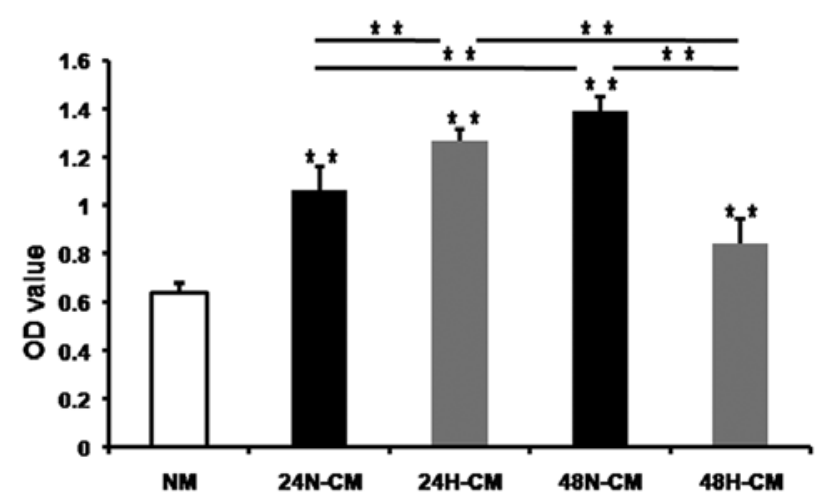

Figure 2. The effects of conditioned medium (CM) collected from hypoxiatreated PC-3 cells on the proliferation of BM-EPCs $\left({ }^{* *} \mathrm{P}<0.01\right.$, respectively) All CM enhanced BM-EPC proliferation compared with normal medium $(\mathrm{NM})$ as a control $\left({ }^{* *} \mathrm{P}<0.01\right.$, respectively).

array to reveal the changes of cytokines in H-CM compared with N-CM. The cytokine profile for each of the CM was performed using three independent biological samples on protein arrays printed with 174 anti-cytokine antibodies in replicate. Relative expression levels of cytokines could be acquired by comparing the signal intensities. Significantly differentially expressed cytokines were selected as following standards: fold-change $>1.3$-fold and $\mathrm{P}<0.05$ in $\mathrm{H}-\mathrm{CM}$ compared with N-CM, and 24H-CM compared with 48H-CM. The results of cluster analysis are shown in Fig. 4. Compared with $24 \mathrm{~N}-\mathrm{CM}$, most cytokines in $24 \mathrm{H}-\mathrm{CM}$ were upregulated, but compared with $48 \mathrm{~N}-\mathrm{CM}$, most cytokines in $48 \mathrm{H}-\mathrm{CM}$ were downregulated. Compared with $24 \mathrm{~N}-\mathrm{CM}$, most cytokines in $48 \mathrm{~N}-\mathrm{CM}$ were upregulated, however, compared with $24 \mathrm{H}-\mathrm{CM}$, most cytokines in $48 \mathrm{H}-\mathrm{CM}$ were downregulated. These data indicated that in the early stage, hypoxia induced expression of most cytokines in PC-3 cells, but in the later stage, hypoxia repressed expression of several cytokines.

Twenty-five types of cytokines were significantly different between $24 \mathrm{H}-\mathrm{CM}$ and $24 \mathrm{~N}-\mathrm{CM}$, and all these factors increased in 24H-CM (Fig. 5A). Eleven types of cytokines were significantly different between $48 \mathrm{H}-\mathrm{CM}$ and $48 \mathrm{~N}-\mathrm{CM}$ (Fig. 5B), and, among them, only 5 factors increased and 6 decreased in $48 \mathrm{H}-\mathrm{CM}$. Twelve types of cytokines were significantly different between $48 \mathrm{H}-\mathrm{CM}$ and $24 \mathrm{H}-\mathrm{CM}$, and, among them, 4 factors increased and 8 decreased in 48H-CM (Fig. 5C). Forty-four types of cytokines were significantly different between $24 \mathrm{~N}-\mathrm{CM}$ and $48 \mathrm{~N}-\mathrm{CM}$, and, among them, 43 factors increased in 48N-CM (Fig. 5D).

Some cytokines increased the expression in $24 \mathrm{H}-\mathrm{CM}$ compared with that in $24 \mathrm{~N}-\mathrm{CM}$, but these changes were not 


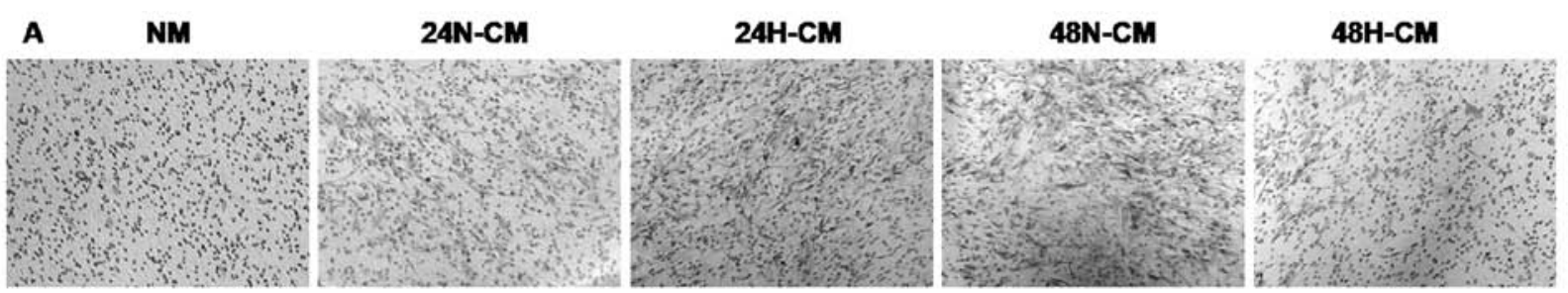

B
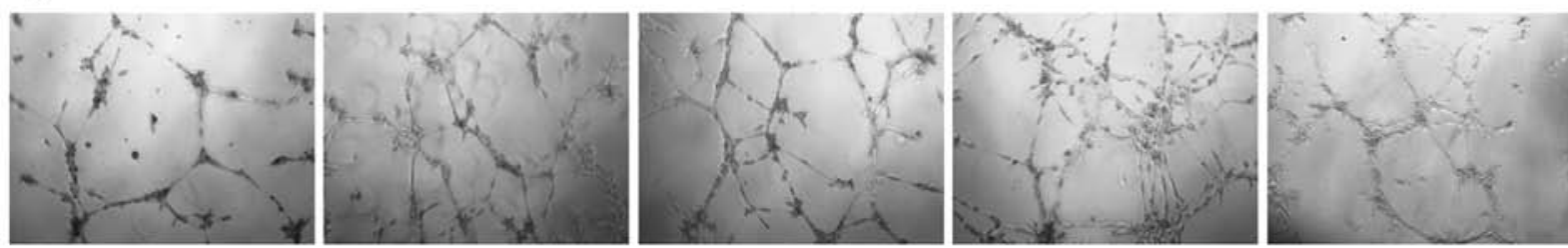

C

D
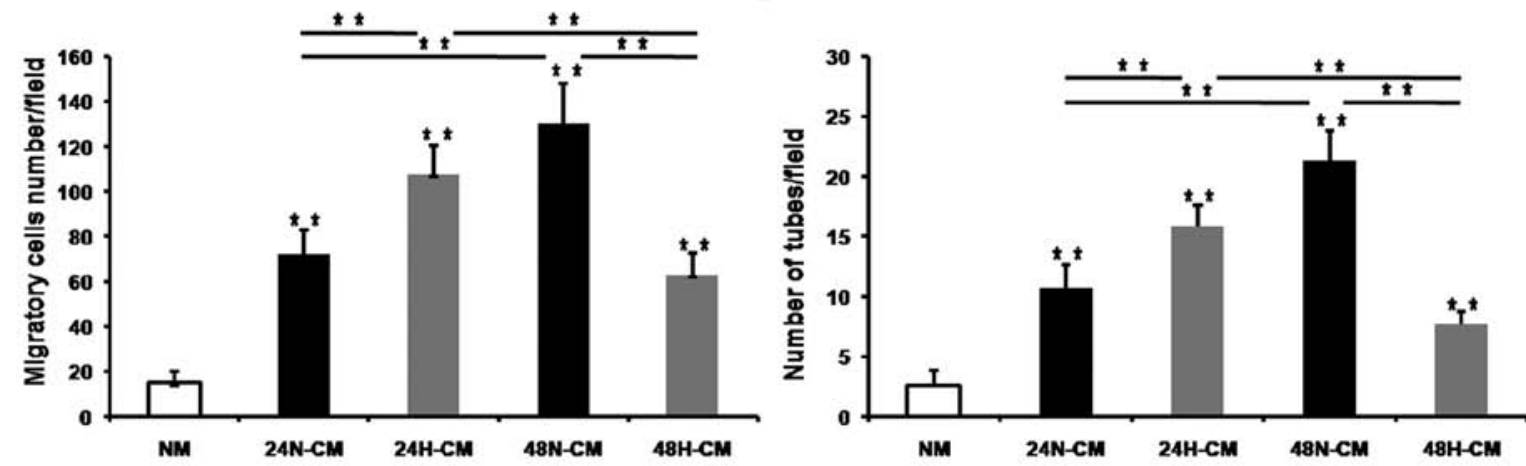

Figure 3. The effects of conditioned medium (CM) collected from hypoxia-treated PC-3 cells on migration and in vitro vasculogenesis of BM-EPCs. All CM enhanced migration and in vitro vasculogenesis of BM-EPCs compared with normal medium (NM) as a control ("P $<<0.01$, respectively). (A and C) Migration capacity of BM-EPCs treated with CM of PC-3 cells ( $\left({ }^{* *} \mathrm{P}<0.01\right.$, respectively). (B and D) In vitro vasculogenesis capacity of BM-EPCs treated with CM of PC-3 cells $(\stackrel{* * *}{P}<0.01$, respectively).

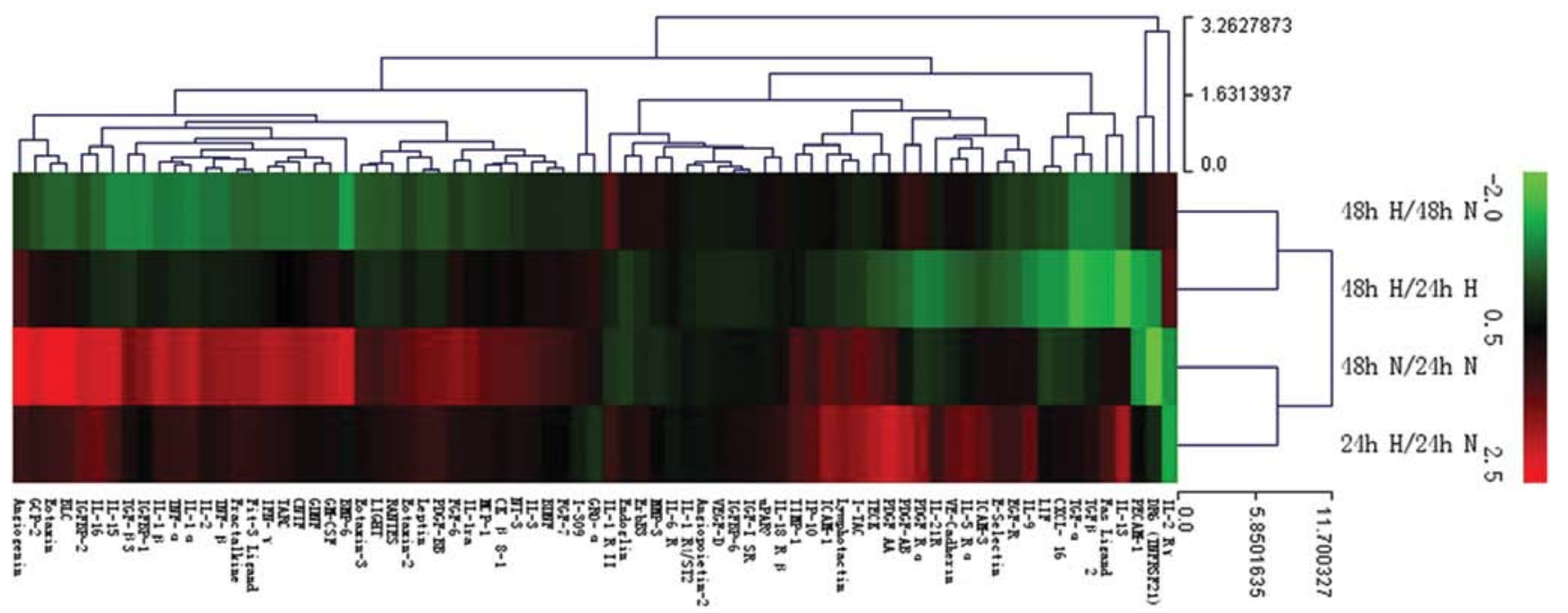

Figure 4. Cluster analyses of all significantly differentially expressed cytokines in hypoxic $(\mathrm{H})$ and normoxic $(\mathrm{N})$ conditioned medium $(\mathrm{CM})$ (fold-change $>1.3$, $\mathrm{P}<0.05$ ).

statistically significant, which play an important role in inducing EPCs homing and vasculogenesis according to the results in literature $(20,23,24)$, including VEGF (fold-change $=2.47$, $\mathrm{P}=0.083$ ), SDF-1 (fold-change $=1.43, \mathrm{P}=0.429$ ), IGF-II (foldchange $=1.84, \mathrm{P}=0.053$ ), $\mathrm{PIGF}$ (fold-change=1.79, $\mathrm{P}=0.125$ ).
Functions of the cytokines with significant alterations in $H$-CM are involved in proliferation, migration and vasculogenesis. Differentially expressed cytokines selected as standards were analyzed by GO analysis. The GO project provides a controlled vocabulary to describe gene and gene 
A

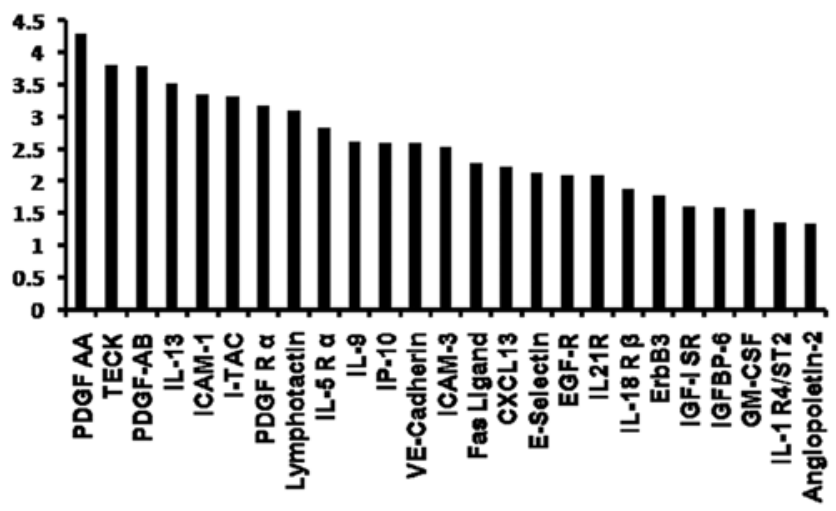

B

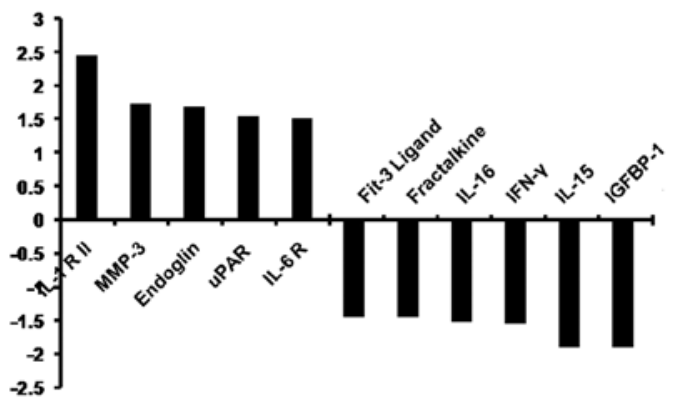

C

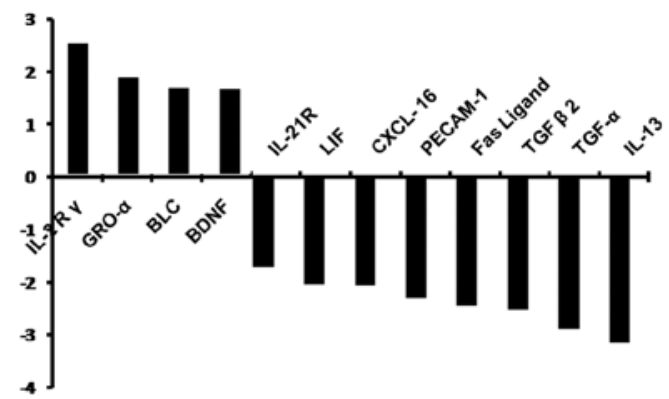

D

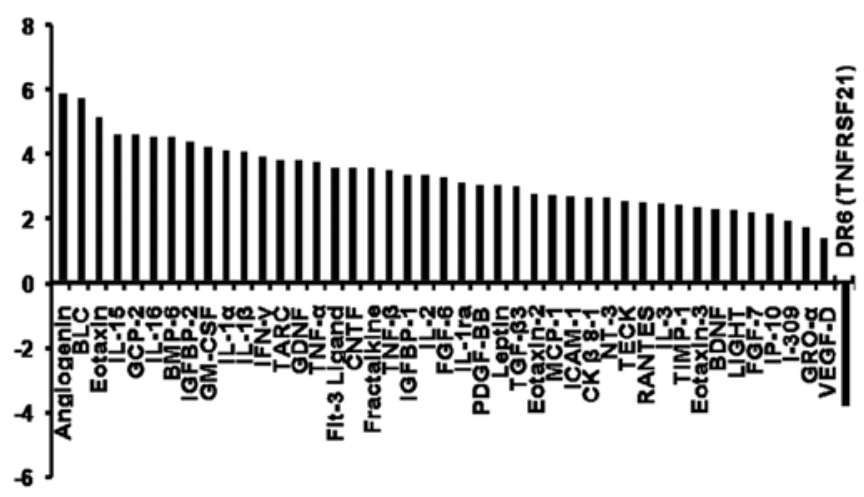

Figure 5. Significantly differentially expressed cytokines in hypoxic $(\mathrm{H})$ and normoxic $(\mathrm{N})$ conditioned medium $(\mathrm{CM})$ (fold-change $>1.3$, $\mathrm{P}<0.05$ ). (A) Changed cytokines in 24-h hypoxia (24H)-CM compared with 24-h normoxia (24N)-CM; (B) changed cytokines in 48H-CM compared with 48N-CM; (C) changed cytokines in 48H-CM compared with $24 \mathrm{H}-\mathrm{CM}$; (D) changed cytokines in 48N-CM compared with $24 \mathrm{~N}-\mathrm{CM}$.

product attributes in any organism (http://www.geneontology. org, GO Result, Fisher's exact method, P-value $\leq 0.05$ ). Therefore, we know the cytokines which may be involved in the change on functions of BM-EPCs by PC-3 CM between hypoxia and normoxia. 'Biological process' and 'molecular function' of those cytokines involved were analyzed and the terms were found, including positive and negative regulation of cell proliferation, chemotaxis, cell mobility, cell migration, vasculogenesis, positive and negative regulation of angiogenesis, angiogenesis, which are involved in the proliferation, migration and tube formation in vitro. GO analysis results showed that several cytokines are involved in proliferation, migration, however, a few cytokines were involved in vasculogenesis and angiogenesis (Table II).

\section{Discussion}

In the present study, we demonstrated that $24 \mathrm{H}-\mathrm{CM}$ of PC- 3 cells of $\mathrm{PCa}$ presented stronger abilities of promoting proliferation and migration, and augmenting in vitro vasculogenesis capacity of BM-EPCs compared to 24N-CM. Moreover, 24H-CM had stronger effects than $48 \mathrm{H}-\mathrm{CM}$. At the same time, our results also demonstrated that hypoxia for $24 \mathrm{~h}$ induced several types of cytokines significantly increasing expression in CM of PC-3 cells. However, downregulated factors were more than upregulated factors in $48 \mathrm{H}-\mathrm{CM}$ compared with $48 \mathrm{~N}-\mathrm{CM}$ or $24 \mathrm{H}-\mathrm{CM}$.
Furthermore, according to the Gene Ontology analysis, all those changed cytokines were involved in the regulation of cell proliferation, chemotaxis, cell motility, cell migration, vasculogenesis and angiogenesis. The changed regularity of cytokines in the $24 \mathrm{H}-\mathrm{CM}$ and $48 \mathrm{H}-\mathrm{CM}$ of PC-3 cells was in concert with the functional changes of BM-EPCs treated with different CM of PC-3 cells in enhancing the proliferation, migration and vasculogenesis potential of BM-EPCs. These data suggest that PCa cells may have the potential to modulate their microenvironment and facilitate BM-EPC migration and vasculogenesis in the early stage of hypoxia.

Our results demonstrated that the function of the majority of the hypoxia-induced cytokines released by PCa cells from bone metastasis in early stage was to promote chemotaxis, cell motility and cell migration. In vivo, as PCa cells colonize in the haematopoietic stem cell niches which are hypoxic (27-29), once into the niches, PCa cells may meet up with the hypoxic bone microenvironment. Those cytokines released by PCa cells in the hypoxic bone microenvironment may recruit BM-EPCs to the parasitizing focus and induce vasculogenesis. Gao et al (4) used mouse models of pulmonary metastasis and identified BM-EPCs as critical regulators of this angiogenic switch. Animal studies showed that BM-EPCs played a role in vasculogenesis within $48 \mathrm{~h}$ after tumor transplantation $(31,32)$. A kinetic analysis of EPC contribution as a function of tumor growth showed that EPCs are recruited to early tumors 
Table II. Gene Ontology analysis results of differentially expressed cytokines in conditioned media which related with proliferation, migration and angiogenesis.

\begin{tabular}{lcccc}
\hline \multicolumn{1}{c}{$24 \mathrm{H} / 24 \mathrm{~N}$} & $48 \mathrm{H} / 48 \mathrm{~N}$ & $48 \mathrm{H} / 24 \mathrm{H}$ & $48 \mathrm{~N} / 24 \mathrm{~N}$ \\
\hline $\begin{array}{l}\text { Positive regulation } \\
\text { of cell proliferation }\end{array}$ & \multicolumn{1}{c}{ Upregulated } & Upregulated & Downregulated & Upregulated \\
(GO:0008284) & FASLG, IGF1, IL9, IL13, & IL6R & FASLG, IL13, LIF, ANG, CCL2, CCL5, CCL11, CCL24, & Downregulated \\
& TGFA, TGFB2 & CCL26, CNTF, CSF2, CXCL10, \\
& PDGFA, PDGFB, PDGFRA & IFNG, IL15 & & FGF7, FGF6, FIGF, IFNG, IL1B, IL2, \\
& & & & IL3, IL15, PDGFB, TIMP1, TNF
\end{tabular}

Negative regulation of cell proliferation (GO:0008285)

Chemotaxis (GO:0006935)
Upregulated ANGPT2, CCL25, CXCL10, CXCL11, CXCL13, EGFR, PDGFA, PDGFB, XCL1

Cell migration (GO:0016477)

Cell motility (GO:0048870)

Vasculogenesis (GO:0001570)

Positive regulation of angiogenesis (GO:0045766)

Negative regulation of angiogenesis (GO:0016525)

\section{Angiogenesis}

(GO:0001525)

\begin{abstract}
Upregulated ANGPT2, CCL25, EGFR, ICAM1, IGF1, PDGFA, PDGFB, PDGFRA, SELE
\end{abstract}

Upregulated
ENG
Downregulated
FNG, IL15

Upregulated

ENG, IL6R,

PLAUR

Downregulated IL16, CX3CL1

\author{
Upregulated \\ ENG, IL6R \\ Downregulated \\ CX3CL1, IL16 \\ Upregulated \\ ENG \\ Downregulated \\ CX3CL1
}
Upregulated CXCL1

Downregulated

LIF, TGFB2

Upregulated

CXCL1, CXCL13 CCL1, CCL2, CCL5, CCL11, CCL17,

Downregulated CXCL1,CXCL6, CXCL10, CXCL13, CXCL16, TGFB2 CX3CL1, FGF7, FIGF, GDNF, IL1B

Downregulated

CXCL16, ECAM1, ANG, CCL2, CCL5, CCL11, CCL24, TGFB2
Upregulated ENG
Downregulated CX3CL1 CCL23, CCL24, CL25, CCL26, IL16, NTF3, PDGFB

\author{
ANG, CCL23, CXCL1, IFNG, IL1A \\ IL1B, IL2, IL15, LTA, TGFB3, TNF
}

Upregulated

Upregulated

CCL25, CCL26, CX3CL1, FGF7, FIGF, GDNF, ICAM1, IL1B, IL16, PDGFB, TNF
Downregulated

FASLG, LIF

FASLG, LIF, TGFB2
Downregulated
Upregulated

ANG, CCL2, CCL11, CCL24,

CX3CL1, FGF6, FIGF, IL1A, IL1B preceding vessel formation, followed by differentiation into endothelial cells and luminal incorporation into a subset of sprouting tumor neovessels (12). These findings suggested that BM-EPCs might contribute to the development of micrometastasis of $\mathrm{PCa}$ in bone marrow in the early stage of colonization of metastatic PCa cells.

The migration of EPCs induced by cytokines released from tumor cells is one of the key mechanisms in the process of vascular development of hypoxia-induced vasculogenesis and one of the rate-limiting steps in the homing of EPCs (10-12). Several studies have demonstrated that factors such as VEGF (20), SDF-1 (20), G-CSF (21), PIGF (23) and IGF2 (24) are pivotal in the regulation of homing of EPCs. In the present study, the results showed that hypoxia did not induce statistically significant high expressions of the above-mentioned cytokines in PC-3 cells.
However, the expression levels of GM-CSF, angiopoietin-2, PDGF-AB, and E-selectin were induced significantly by 24-h hypoxia and 2 types of cytokines, endoglin (ENG) and IL6, in 48-h hypoxia. Previous studies have demonstrated that GM-CSF (22), E-selectin (25), angiopoietin-2 (26), PDGF-AB (33), ENG (34) and IL6 (5) are the important factors for the hypoxia-induced homing of BM-EPCs in the vasculogenesis process. Therefore, we speculated that these cytokines might play a more important role in the hypoxiainduced homing of EPCs in PCa bone metastasis compared to VEGF, SDF-1, G-CSF, PIGF and IGF2. We also found that the expression levels of several cytokines significantly upregulated, including CXCL13 (BLC), CXCL10 (IP-10), CCL25 (TECK), CXCL11 (I-TAC), lymphotactin (XCL1), ICAM1, EGFR, IGF1R in CM of PC-3 cells by $24-\mathrm{h}$ hypoxia. These cytokines are involved in chemotaxis, cell motility and cell 
migration according to the gene ontology analysis. Some of them have also been proved to be involved in EPC homing, such as ICAM1 (36,37), IGF1R (38). Those cytokines may be other factors that recruit EPCs to the tumor. Therefore, EPC homing might be a combined effect of several cytokines in the vasculogenesis process of bone metastasis of $\mathrm{PCa}$.

EPCs that are incorporated into the tumor vasculature could constitute as much as $50 \%$ (39), but it is not known if the proliferating capabilities of EPCs are controlled by the tumor microenvironment factors. In the present study, the results showed that CM of PC-3 cells by 24-h hypoxia promoted EPC proliferation and hypoxia significantly induced PC-3 cells releasing several cytokines of positive regulation of cell proliferation. According to the Gene Ontology analysis, those cytokines included GM-CSF, IL5, IL13, EGFR, IGF1R, IL9, CXCL10 in 24-h hypoxia, and IFNG, IL15, IL6 in 48-h hypoxia, among them, GM-CSF (40) was proved to have the function of promoting proliferation of EPCs. Some of those cytokines have also been reported to be involved in EPC homing, such as GM-CSF (22), IL6 (35) and IGF1R (38). Therefore, the proliferating capabilities of EPCs may be controlled by the tumor microenvironment.

Our results showed that $24 \mathrm{H}-\mathrm{CM}$ of PC-3 cells augmented the in vitro vasculogenesis capacity of BM-EPCs and hypoxia significantly induced PC-3 cells releasing angiopoietin-2. Since angiopoietin-2 was proved to promote vasculogenesis of EPC (41), it was suggested that angiopoietin-2 might play an important role in the vasculogenesis of BM-EPCs in PCa progression. The results also showed that ENG and IL6 significantly increased in 48H-CM of PC-3 cells and ENG (34) and IL6 (35) have been proved to be involved in vasculogenesis. It was suggested that ENG and IL6 might play a role in vasculogenesis in 48-h hypoxia.

In conclusion, our study detected 174 cytokines in $\mathrm{CM}$ of PC-3 cells from bone metastasis of PCa and found that 24-h hypoxia significantly induced the secretion of several cytokines. Those released cytokines in the CM of PC-3 cells by 24-h hypoxia promoted proliferation and migration and augmented vasculogenesis in vitro of BM-EPCs. Our data suggested that PCa cells may have the potential to modulate their microenvironment and facilitate BM-EPC migration and vasculogenesis in the early stage of hypoxia and BM-EPCs might contribute to the development of micrometastasis of PCa in bone marrow in the early stage of colonization of metastatic PCa cells. However, aside from the cytokines found in this study, other cytokines may be involved in these processes. Furthermore, it also is necessary to detect the functions of these cytokines in future studies. The characterization of tumor-associated BM-EPCs may provide valuable insight for more specific antiangiogenesis therapy and/or tumor diagnosis.

\section{Acknowledgements}

The authors thank Dr Wenjian Wang and Dr Wen Li from the Surgical Laboratory at the First Affiliated Hospital of Sun Yat-sen University for their help. We also thank the NSFCGuangdong Joint funding, China (no. u0732001); the Science and Technology planning project of Guangdong Province, China (no. 2008B030301037), the Science and Technology Planning Project of Guangzhou, China (11C22060772).

\section{References}

1. Roodman GD: Mechanisms of bone metastasis. N Engl J Med 350: 1655-1664, 2004.

2. Mundy GR: Metastasis to bone: causes, consequences and therapeutic opportunities. Nat Rev Cancer 2: 584-593, 2002.

3. Weidner N, Semple JP, Welch WR and Folkman J: Tumor angiogenesis and metastasis - correlation in invasive breast carcinoma. N Engl J Med 324: 1-8, 1991.

4. Gao D, Nolan DJ, Mellick AS, Bambino K, McDonnell K and Mittal V: Endothelial progenitor cells control the angiogenic switch in mouse lung metastasis. Science 319: 195-198, 2008.

5. Liao D and Johnson RS: Hypoxia: a key regulator of angiogenesis in cancer. Cancer Metastasis Rev 26: 281-290, 2007.

6. Lee SL, Rouhi P, Dahl Jensen L, et al: Hypoxia-induced pathological angiogenesis mediates tumor cell dissemination, invasion, and metastasis in a zebrafish tumor model. Proc Natl Acad Sci USA 106: 19485-19490, 2009.

7. Senger DR and Davis GE: Angiogenesis. Cold Spring Harb Perspect Biol 3: a005090, 2011.

8. Asahara T and Kawamoto A: Endothelial progenitor cells for postnatal vasculogenesis. Am J Physiol Cell Physiol 287: C572-C579, 2004.

9. Wickersheim A, Kerber M, de Miguel LS, Plate KH and Machein MR: Endothelial progenitor cells do not contribute to tumor endothelium in primary and metastatic tumors. Int $\mathrm{J}$ Cancer 125: 1771-1777, 2009.

10. De Palma M and Naldini L: Role of haematopoietic cells and endothelial progenitors in tumour angiogenesis. Biochim Biophys Acta 1766: 159-166, 2006.

11. Spring H, Schüler T, Arnold B, Hämmerling GJ and Ganss R: Chemokines direct endothelial progenitors into tumor neovessels. Proc Natl Acad Sci USA 102: 18111-18116, 2005.

12. Nolan DJ, Ciarrocchi A, Mellick AS, et al: Bone marrow-derived endothelial progenitor cells are a major determinant of nascent tumor neovascularization. Genes Dev 21: 1546-1558, 2007.

13. Gao D, Nolan D, McDonnell K, Vahdat L, Benezra R, Altorki N and Mittal V: Bone marrow-derived endothelial progenitor cells contribute to the angiogenic switch in tumor growth and metastatic progression. Biochim Biophys Acta 1796: 33-40, 2009.

14. Martin-Padura I, Gregato G, Marighetti $P$, et al: The white adipose tissue used in lipotransfer procedures is a rich reservoir of $\mathrm{CD} 34^{+}$progenitors able to promote cancer progression. Cancer Res 72: 325-334, 2012.

15. Döme B, Hendrix MJ, Paku S, Tóvári J and Tímár J: Alternative vascularization mechanisms in cancer: pathology and therapeutic implications. Am J Pathol 170: 1-15, 2007.

16. Strohmeyer D, Rössing C, Strauss F, Bauerfeind A, Kaufmann O and Loening S: Tumor angiogenesis is associated with progression after radical prostatectomy in $\mathrm{pT} 2 / \mathrm{pT} 3$ prostate cancer. Prostate 42: 26-33, 2000.

17. Revelos K, Petraki C, Scorilas A, et al: Correlation of androgen receptor status, neuroendocrine differentiation and angiogenesis with time-to-biochemical failure after radical prostatectomy in clinically localized prostate cancer. Anticancer Res 27: 3651-3660, 2007.

18. Lissbrant IF, Stattin P, Damber JE and Bergh A: Vascular density is a predictor of cancer-specific survival in prostatic carcinoma. Prostate 33: 38-45, 1997.

19. Asahara T, Takahashi T, Masuda H, et al: VEGF contributes to postnatal neovascularization by mobilizing bone marrowderived endothelial progenitor cells. EMBO J 18: 3964-3972, 1999.

20. Orimo A, Gupta PB, Sgroi DC, et al: Stromal fibroblasts present in invasive human breast carcinomas promote tumor growth and angiogenesis through elevated SDF-1/CXCL12 secretion. Cell 121: 335-348, 2005.

21. Powell TM, Paul JD, Hill JM, et al: Granulocyte colony-stimulating factor mobilizes functional endothelial progenitor cells in patients with coronary artery disease. Arterioscler Thromb Vasc Biol 25: 296-301, 2005.

22. Takahashi T, Kalka C, Masuda H, et al: Ischemia- and cytokineinduced mobilization of bone marrow-derived endothelial progenitor cells for neovascularization. Nat Med 5: 434-438, 1999.

23. Li B, Sharpe EE, Maupin AB, Teleron AA, Pyle AL, Carmeliet P and Young PP: VEGF and PIGF promote adult vasculogenesis by enhancing EPC recruitment and vessel formation at the site of tumor neovascularization. FASEB J 20: 1495-1497, 2006. 
24. Maeng YS, Choi HJ, Kwon JY, et al: Endothelial progenitor cell homing: prominent role of the IGF2-IGF2R-PLC $\beta 2$ axis. Blood 113: 233-243, 2009.

25. Liu ZJ, Tian R, Li Y, An W, Zhuge Y, Livingstone AS and Velazquez OC: Inhibition of tumor angiogenesis and melanoma growth by targeting vascular E-selectin. Ann Surg 254: 450-457, 2011.

26. Gill KA and Brindle NP: Angiopoietin-2 stimulates migration of endothelial progenitors and their interaction with endothelium. Biochem Biophys Res Commun 336: 392-396, 2005.

27. Shiozawa Y, Pedersen EA, Havens AM, et al: Human prostate cancer metastases target the hematopoietic stem cell niche to establish footholds in mouse bone marrow. J Clin Invest 121: 1298-1312, 2011.

28. Chow DC, Wenning LA, Miller WM and Papoutsakis ET: Modeling $\mathrm{pO}(2)$ distributions in the bone marrow hematopoietic compartment. II. Modified Kroghian models. Biophys J 81 685-696, 2001.

29. Harrison JS, Rameshwar P, Chang V and Bandari P: Oxygen saturation in the bone marrow of healthy volunteers. Blood 99 394, 2002.

30. Tang Y, Huang B, Sun L, Peng X, Chen X and Zou X: Ginkgolide B promotes proliferation and functional activities of bone marrow-derived endothelial progenitor cells: involvement of Akt/eNOS and MAPK/p38 signaling pathways. Eur Cell Mater 21: 459-469, 2011.

31. Asahara T, Masuda H, Takahashi T, et al: Bone marrow origin of endothelial progenitor cells responsible for postnatal vasculogenesis in physiological and pathological neovascularization. Circ Res 85: 221-228, 1999.

32. Lyden D, Hattori K, Dias S, et al: Impaired recruitment of bonemarrow-derived endothelial and hematopoietic precursor cells blocks tumor angiogenesis and growth. Nat Med 7: 1194-1201, 2001.
33. Langer HF, May AE, Vestweber D, De Boer HC, Hatzopoulos AK and Gawaz M: Platelet-induced differentiation of endothelial progenitor cells. Semin Thromb Hemost 33: 136-143, 2007.

34. Nassiri F, Cusimano MD, Scheithauer BW, et al: Rotondo F Endoglin (CD105): a review of its role in angiogenesis and tumor diagnosis, progression and therapy. Anticancer Res 31: 2283-2290, 2011.

35. Fan Y, Ye J, Shen F, et al: Interleukin-6 stimulates circulating blood-derived endothelial progenitor cell angiogenesis in vitro. J Cereb Blood Flow Metab 28: 90-98, 2008.

36. Silverman MD, Haas CS, Rad AM, Arbab AS and Koch AE: The role of vascular cell adhesion molecule 1/ very late activation antigen 4 in endothelial progenitor cell recruitment to rheumatoid arthritis synovium. Arthritis Rheum 56: 1817-1826, 2007.

37. Wu Y, Ip JE, Huang J, et al: Essential role of ICAM-1/CD18 in mediating EPC recruitment, angiogenesis, and repair to the infarcted myocardium. Circ Res 99: 315-322, 2006.

38. Humpert PM, Djuric Z, Zeuge U, et al: Insulin stimulates the clonogenic potential of angiogenic endothelial progenitor cells by IGF-1 receptor-dependent signaling. Mol Med 14: 301-308, 2008.

39. Garcia-Barros M,Paris F, Cordon-Cardo C, et al: Tumor response to radiotherapy regulated by endothelial cell apoptosis. Science 300: 1155-1159, 2003

40. Wang QR, Wang F, Zhu WB, Lei J, Huang YH, Wang BH and Yan Q: GM-CSF accelerates proliferation of endothelial progenitor cells from murine bone marrow mononuclear cells in vitro. Cytokine 45: 174-178, 2009.

41. Kim KL, Shin IS, Kim JM, et al: Interaction between Tie receptors modulates angiogenic activity of angiopoietin 2 in endothelial progenitor cells. Cardiovasc Res 72: 394-402, 2006. 